\title{
AOCS OPERATIONS PREPARATION FOR THE BEPICOLOMBO MISSION TO MERCURY
}

\author{
C. Steiger ${ }^{1}$, A. Altay $^{2}$, E. Montagnon ${ }^{1}$, and V. Companys ${ }^{1}$ \\ ${ }^{1} \mathrm{ESA} / \mathrm{ESOC}$ \\ 5 Robert-Bosch Str., Darmstadt 64293, Germany \\ ${ }^{2}$ Terma GmbH \\ 5 Robert-Bosch Str., Darmstadt 64293, Germany
}

The 2018 ESA/JAXA BepiColombo mission to Mercury features a complex modular design, with two scientific Mercury orbiters and a cruise module. The spacecraft $(\mathrm{S} / \mathrm{C})$ and mission design lead to a number of challenges for the attitude and orbit control system (AOCS), including electric propulsion usage during cruise to Mercury, AOCS capability to deal with several S/C configurations, and strict attitude constraints owing to the harsh thermal environment. This paper presents the activities for AOCS operations preparation by ESA/ESOC, covering the current preparation status as well as an outlook on upcoming activities before launch.

\section{ACRONYM LIST}

$\begin{array}{ll}\text { AIV } & \text { Assembly, Integration, and Verification } \\ \text { AOCS } & \text { Attitude and Orbit Control System } \\ \text { APME } & \text { Antenna Pointing Mechanism Electronics (for High Gain and } \\ & \text { Medium Gain Antenna) } \\ \text { ASD } & \text { Airbus Defence and Space } \\ \text { BMCS } & \text { BepiColombo Mission Control System } \\ \text { CPS } & \text { Chemical Propulsion System } \\ \text { EEPROM } & \text { Electrically Erasable Programmable Read-Only Memory } \\ \text { EPCM } & \text { Electric Propulsion Control Mode } \\ \text { ESP } & \text { Earth Strobing Phase } \\ \text { ETB } & \text { Engineering Test Bed } \\ \text { FCE } & \text { Failure Control Electronics } \\ \text { FCT } & \text { Flight Control Team } \\ \text { FDIR } & \text { Failure Detection, Isolation, and Recovery } \\ \text { FOP } & \text { Flight Operations Plan }\end{array}$

(C) The Authors, published by EDP Sciences. This is an open access article distributed under the terms of the Creative Commons Attribution License 4.0 (http://creativecommons.org/licenses/by/4.0/). 


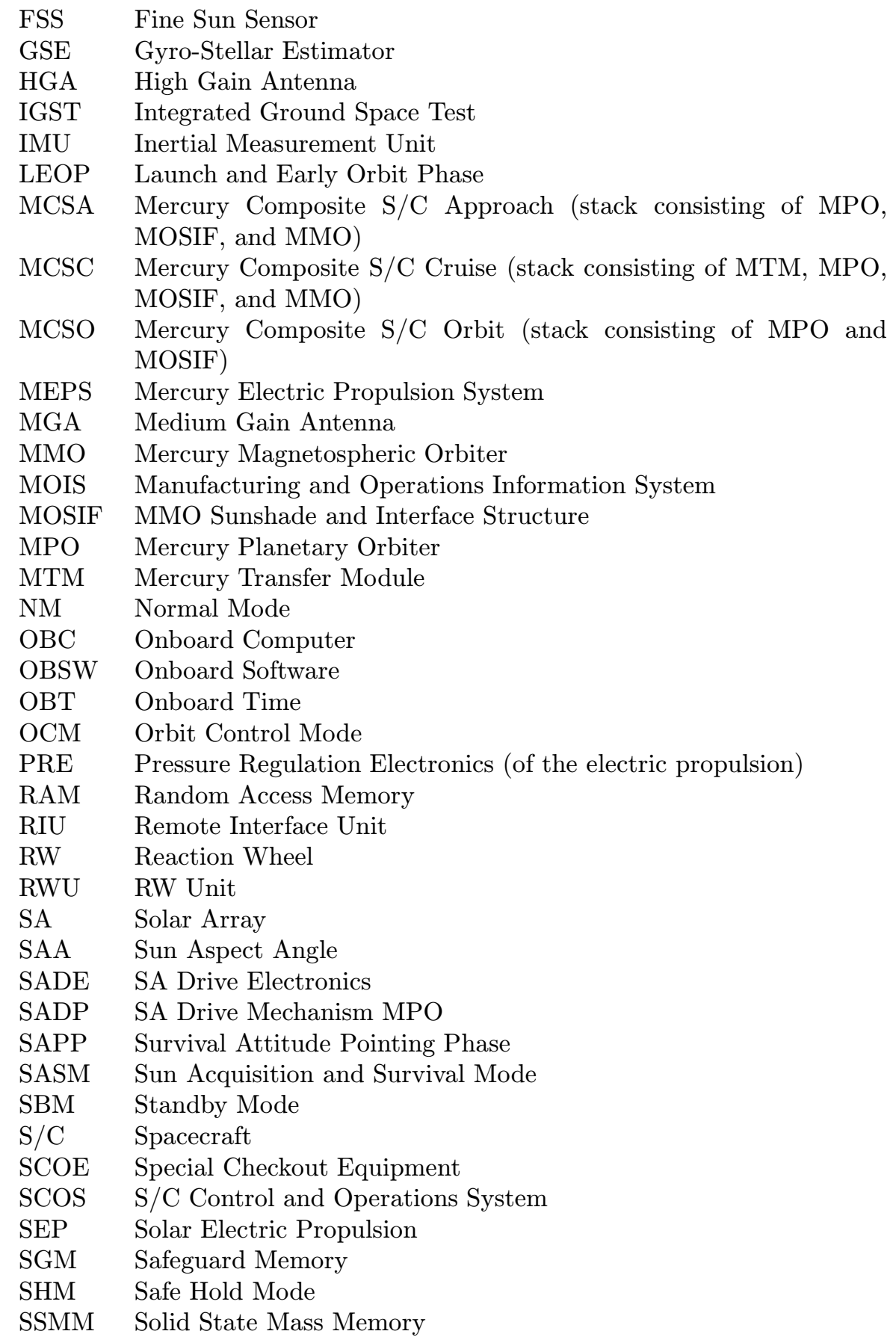


STAP Star Acquisition Phase

STR Star Tracker

SVT System Validation Test

TCP Thruster Control Phase

TPE Thruster Pointing Electronics (of the electric propulsion)

TPM Thruster Pointing Mechanism (of the electric propulsion)

UTC Coordinated Universal Time

WCP Wheel Control Phase

\section{INTRODUCTION}

\subsection{Mission Overview}

BepiColombo, an ESA cornerstone mission to Mercury in collaboration with the Japanese Space Agency (JAXA), will study the planet interior and surface and its environment, i. e., both exosphere and magnetosphere. The mission consists of ESA's Mercury Planetary Orbiter (MPO) and JAXA's Mercury Magnetospheric Orbiter (MMO), launched together as a single composite that includes a dedi-

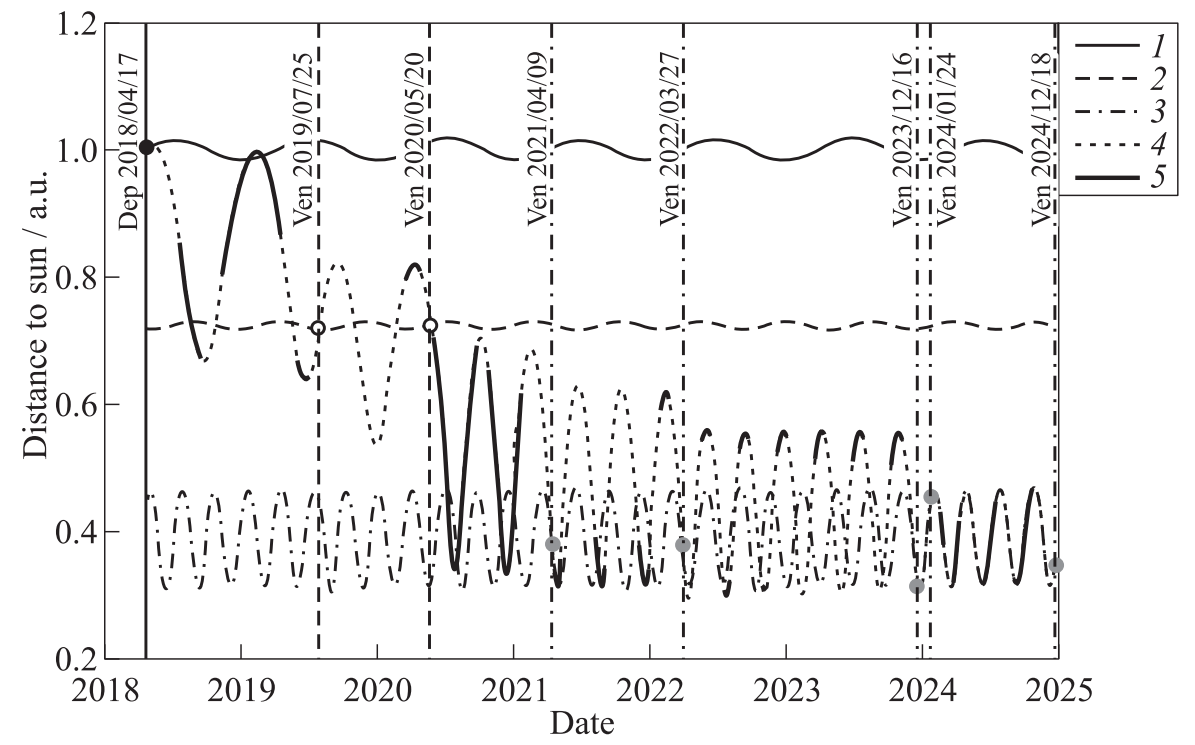

Figure 1 Interplanetary cruise trajectory for April 2018 launch, showing Sun distance, electric propulsion usage ("SEP"), and planetary flybys at Venus and Mercury: 1 Earth; 2 - Venus; 3 - Mercury; 4 - Coast; and 5 - SEP 
cated propulsion module (Mercury Transfer Module, MTM). Mercury Planetary Orbiter and MTM are developed under ESA contract by an international consortium led by Airbus Defence and Space Germany.

BepiColombo is planned to be launched in 2018 with Ariane- 5 from Kourou. The launch will be followed by a 6.5 -year cruise phase, including planetary swingbys at Venus and Mercury, eventually achieving a weak capture by Mercury in late 2024 (Fig. 1). During the cruise phase, electric propulsion will be used for extended periods of time. This is provided by the MTM module, which will be jettisoned at Mercury arrival. A set of complex manoeuvres will deliver the MMO to its operational orbit and, finally, the MPO will be put into its $1500 \times 480 \mathrm{~km}$ polar orbit (orbital period of $2.2 \mathrm{~h}$ ) to perform its scientific mission for one Earth year, with 1 year extension possible [1].

\subsection{The BepiColombo Spacecraft}

Figure 2 shows an artist's impression of BepiColombo. The combined stack can have the following configurations:

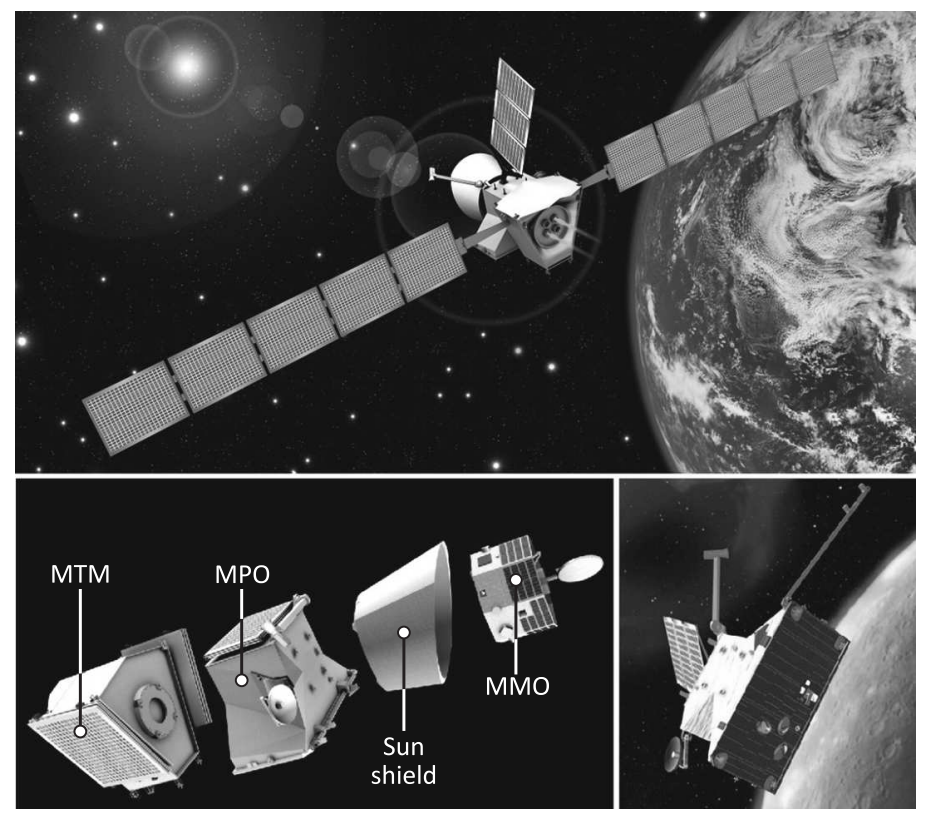

Figure 2 Artist's impression of the BepiColombo spacecraft in cruise configuration (top) with the various elements of the cruise stack in exploded view (bottom left) and the MPO at Mercury (bottom right) 
- Mercury Composite S/C Cruise (MCSC): MTM, MPO, MMO sunshade and interface structure (MOSIF), and MMO;

- Mercury Composite S/C Approach (MCSA): MPO, MOSIF, and MMO following separation of the MTM; and

- Mercury Composite S/C Orbit (MCSO): MPO and MOSIF following release of the MMO.

During interplanetary cruise, the MMO is a passive passenger, i. e., the necessary resources and services are provided by the MPO until it is delivered into its mission orbit. The MTM provides solar electric propulsion (SEP) and all services not required in Mercury orbit.

The MPO accommodates 11 scientific instruments and has a box-like shape with a size of $3.9 \times 2.2 \times 1.7 \mathrm{~m}$ and a dry mass of about $1080 \mathrm{~kg}$. The harsh thermal environment around Mercury imposes strong requirements on the S/C design, requiring high-temperature multilayer insulation and solar array (SA) technology. A radiator to dump excess heat into space is mounted on one side of the S/C which may not be exposed to Sun or Mercury.

The MPO 3-axis stabilized AOCS consists of star trackers, inertial measurement units (IMUs), fine Sun sensors, reaction wheels (RWs), and chemical propulsion. The AOCS design is driven by the challenging thermal environment around Mercury, requiring special guidance profiles for the MPO SA (to avoid overheating) and rapid $\mathrm{S} / \mathrm{C}$ attitude stabilization in case of contingencies. In order to cope with these contingency cases, the onboard computer (OBC) contains a separate processing unit, namely, the Failure Control Electronics (FCE), for controlling the $\mathrm{S} / \mathrm{C}$ attitude in case of transient unavailability of the main OBC. For deep space communications, the MPO uses a X/Ka-band deep space transponder with steerable high gain (HGA) and medium gain antennae (MGA).

In addition to dual mode bipropellant chemical propulsion, the MTM features a SEP system with 4 steerable thrusters based on the Kaufman-type electric bombardment ion motor (maximal thrust $145 \mathrm{mN}$ ). The high power demand by the MTM electric propulsion (up to $11 \mathrm{~kW}$ ) is satisfied with large SAs (area of over $40 \mathrm{~m}^{2}$ in total) using the same high-temperature technology as for the MPO.

\subsection{The BepiColombo Mission Operations Centre at ESA/ESOC}

Operations of the composite $\mathrm{S} / \mathrm{C}$ and the MPO will be conducted from the European Space Operations Centre (ESOC) in Darmstadt, Germany. The main elements of the BepiColombo Mission Operations Centre (BCMOC) at ESOC are shown in Fig. 3. It features the typical setup for ESA/ESOC deep space missions, including a SCOS-2000 based mission control system, a standalone 


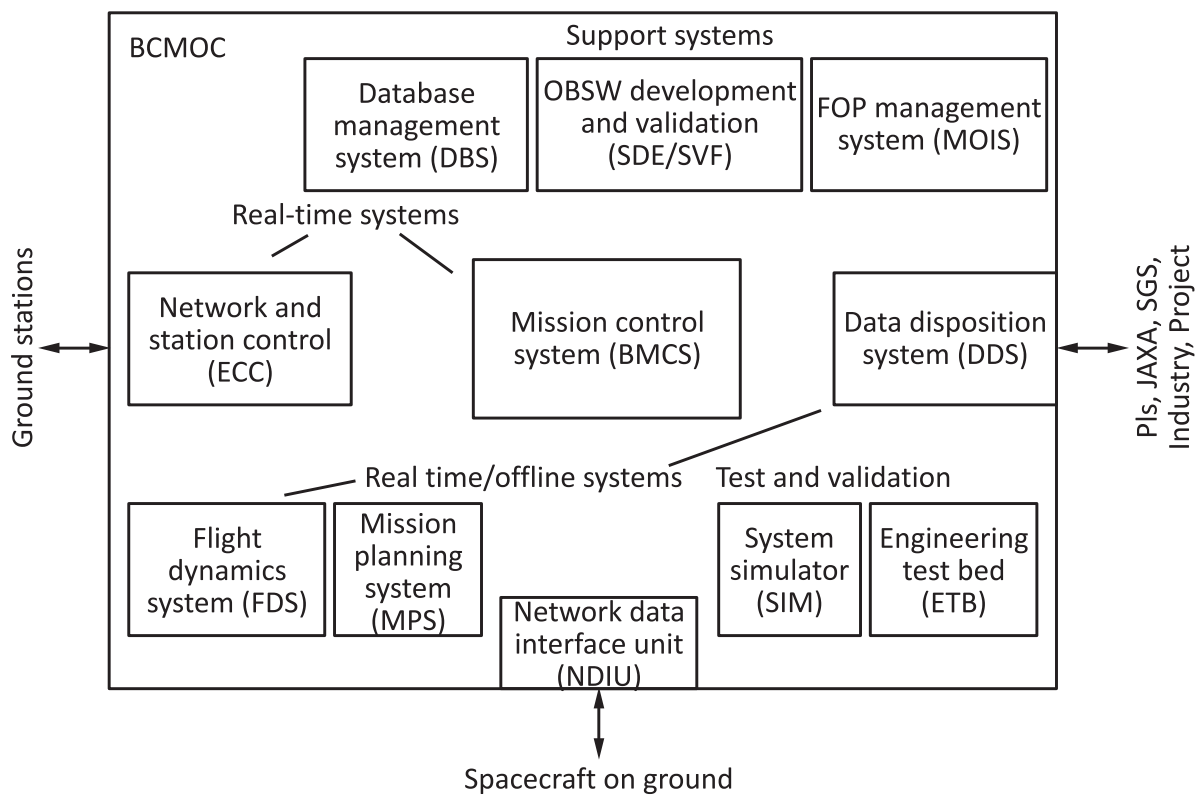

Figure 3 Main elements of the BepiColombo ground segment at ESOC. The ETB will be delivered from industry to ESOC after the launch (SDE/SVF - software development environment/software validation facility; ECC - ESA Tracking Network (ESTRACK) Control Centre)

mission planning system, and a SIMSAT-based S/C simulator. The simulator, running the MPO flight onboard software (OBSW) on a processor emulator, is a key tool for operations preparation [2].

The Flight Control Team (FCT) performs the operations of BepiColombo and interfaces with various multimission support groups at ESOC, such as flight dynamics, ground segment software and hardware support (e.g., mission control system), and ground station operations. For deep space missions, there is a particularly close relation to the flight dynamics team due to the complex navigation and AOCS operations activities.

Prior to launch, the FCT deals with all aspects of operations preparation. Key activities include the following:

- specification and acceptance testing of mission control system, planning system, and simulator;

- preparation of operational products: writing of the Flight Operations Plan (FOP) based on inputs provided by the prime $\mathrm{S} / \mathrm{C}$ contractor as well as population of the $\mathrm{S} / \mathrm{C}$ database; and 
- execution of tests with the S/C flight model as well as the engineering test bed (ETB), with the prime objective of validating the ESOC ground segment and operational products.

Due to the highly constrained mission and complex configuration of the composite $\mathrm{S} / \mathrm{C}$, BepiColombo operations are very challenging [3].

\section{THE ATTITUDE AND ORBIT CONTROL SYSTEM}

Although BepiColombo shares many commonalities with AOCS design from previous ESA interplanetary mission like Rosetta, Venus Express, and Mars Express, the following peculiarities make the BepiColombo AOCS particularly challenging:

- S/C modularity: the AOCS has to deal with various composite S/C configurations, with significantly different $\mathrm{S} / \mathrm{C}$ properties and several sensors and actuators duplicated on MTM and MPO. The S/C attitude in survival and safe mode differs depending on the composite S/C configuration;

- initial attitude acquisition in safe mode: in the harsh thermal environment at Mercury, there would be no time for the AOCS to perform an attitude acquisition from "lost in space" conditions (e.g., by performing a Sun search using Sun sensors) at safe mode entry. Instead, the last known S/C attitude and dynamics are continuously stored in safeguard memory (SGM) and then are retrieved in case of safe mode entry. Another consequence of the severe attitude constraints is that at safe mode entry, when the $\mathrm{S} / \mathrm{C}$ is not controlled by the prime $\mathrm{OBC}$ due to a reboot, the FCE is taking over control for a few minutes, running a simplified AOCS software able to damp S/C angular rates and ensure correct Sun pointing;

- solar array control: owing to technological limitations, the MPO SA cannot be pointed directly to the Sun. Instead, it has to be off pointed such that sufficient power is obtained, yet maximum temperature limits are not violated. This is achieved by ground-provided polynomial SA steering profiles continuously adjusting the SA position throughout the MPO's orbit around Mercury;

- attitude and solar array guidance for safe mode: correct attitude and SA pointing after safe mode entry is crucial for S/C survival. As the orbit around Mercury is left to drift and the margins available are very limited, guidance for safe mode requires frequent updates (about once per week). An error in the updated guidance parameter setting could lead to an end of the mission; and 


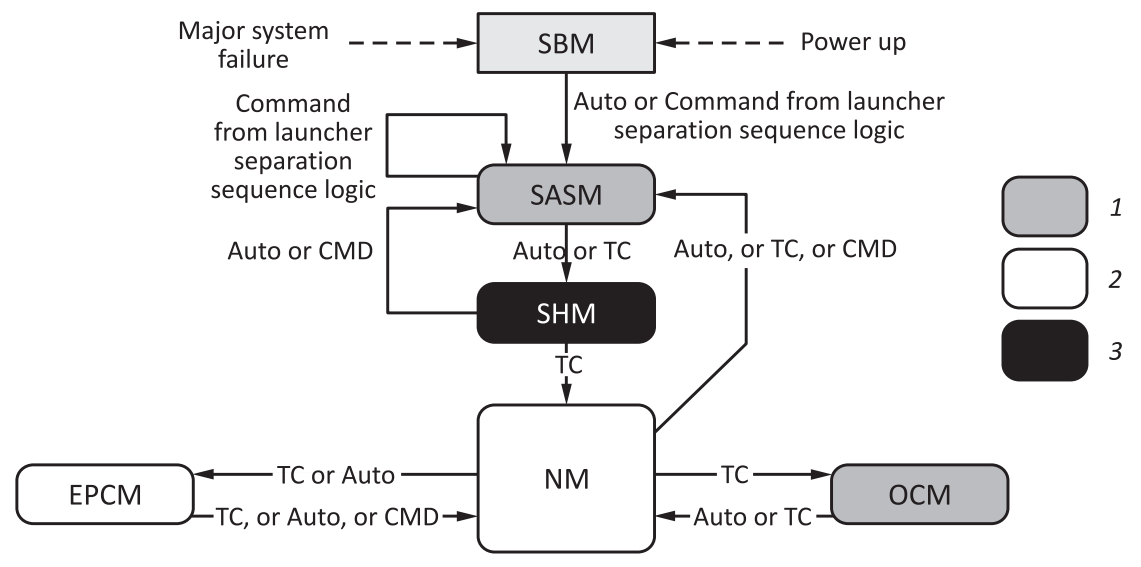

Figure 4 AOCS modes overview: 1 - mode controlled with thrusters; 2 - mode controlled with wheels; 3 - mode controlled with thrusters or wheels; TC - ground commanded mode transition; Auto - autonomous mode transition triggered by AOCS; and CMD - autonomous mode transition triggered by data management system (e. g., due to need to reduce power consumption of AOCS)

- electric propulsion: BepiColombo is the first ESA interplanetary mission using electric propulsion.

Figure 4 gives an overview of AOCS modes and mode transitions:

- Standby Mode (SBM): the AOCS is inactive with all units switched off. It is used for ground testing and is only entered transiently during flight (e.g., after safe mode entry);

- Sun Acquisition and Survival Mode (SASM): ultimate backup mode ensuring $\mathrm{S} / \mathrm{C}$ survival in case of major onboard contingencies. Attitude control with thrusters only. Initially, the $\mathrm{S} / \mathrm{C}$ is Sun pointed purely based on ground-provided Sun ephemerides and the last known attitude (propagated in the future by IMU measurements), while Sun sensors and then star trackers are brought into the control loop later on. In SASM, the S/C rotates around the Sun line (in synch with the orbital motion around Mercury when in MPO configuration), pointing the MGA such that it sweeps over the Earth once per revolution;

- Safe Hold Mode (SHM): the S/C is pointed according to ground-provided polynomial profiles. At mode entry, attitude control is done with thrusters, while later RWs are brought into the loop. This is the highest mode that may be entered autonomously following $\mathrm{S} / \mathrm{C}$ safe mode entry. The 
MGA is pointed permanently to Earth based on ground-provided Earth ephemerides;

- Normal Mode (NM): nominal operating mode. Attitude estimation and control as in the later stages of SHM. The steerable HGA is used for communications;

- Orbit Control Mode (OCM): mode for performing trajectory correction manoeuvres using chemical propulsion. Attitude estimation as in NM.

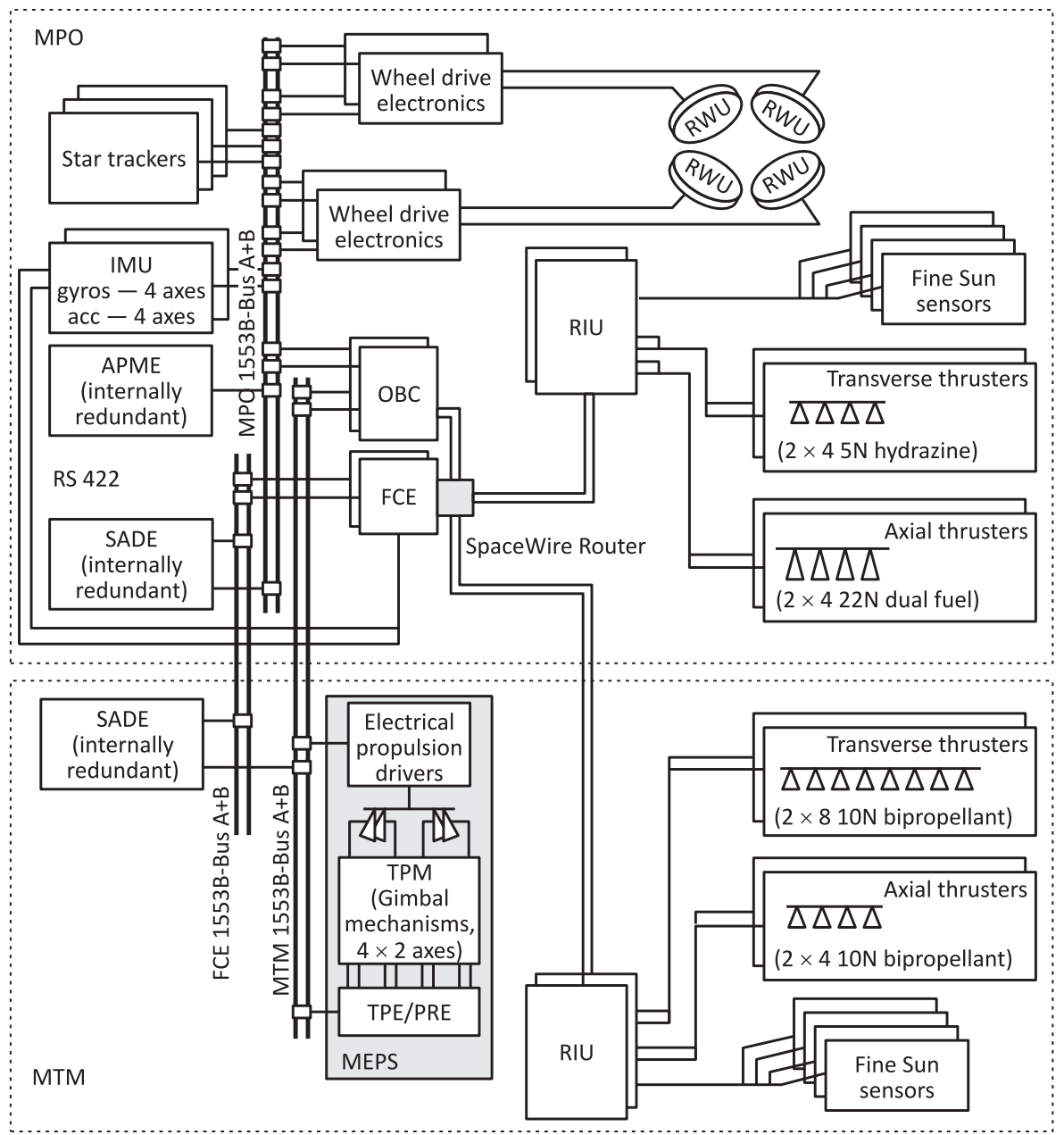

Figure 5 AOCS hardware architecture 
Attitude control performed with thrusters, while RWs are kept at constant speeds; and

- Electric Propulsion Control Mode (EPCM): mode for electric propulsion usage (MCSC configuration only). Attitude control and estimation as in NM.

For what concerns Failure Detection, Isolation, and Recovery (FDIR), the AOCS employs a layered FDIR concept with sets of local, functional, and global surveillances, aiming at isolating a failure rapidly with the least reconfiguration overhead.

Figure 5 gives an overview of the various MPO and MTM units used by the AOCS. The OBSW implementing all AOCS tasks is running on the MPO OBC, controlling all MPO and MTM equipment.

\section{AOCS OPERATIONS PREPARATION AT ESA/ESOC}

\subsection{AOCS Operations Preparation Overview}

As for other subsystems, AOCS operations preparation by the FCT starts well before launch. The following are the most important preparation activities:

- preparation of operational products: AOCS flight operations procedures are written based on input from the $\mathrm{S} / \mathrm{C}$ manufacturer and later validated against the ESOC simulator and the $\mathrm{S} / \mathrm{C}$ in dedicated test slots. The industry-provided S/C database is refined and augmented with operationsspecific information such as the generation of displays, instantiation of specific TCs, transfer module parameter out of limits, etc.;

- validation of the interface to flight dynamics: the flight dynamics system includes the functionalities for orbit determination, S/C dynamics monitoring, and S/C AOCS-specific commands. Commanding products prepared by flight dynamics cover a wide range of activities, for instance, guidance for normal and safe modes, RW momentum management, orbit control manoeuvres with chemical propulsion, solar electric propulsion operations, etc. The required precise set of requests is iterated between the FCT and the flight dynamics team. For BepiColombo, the commanding interface is complex and its validation constitutes a major part of AOCS operations preparation; and

- testing with the flight model and engineering model: prior to launch, ESOC has been granted 30 days to perform System Validation Tests (SVT) with 
the Flight Model. In addition, ESOC can also access the ETB located at the premises of the S/C manufacturer for 30 days to carry out Integrated Ground Space Tests (IGST). Testing activities are carefully prepared and iterated with the S/C manufacturer and the project team at ESA/ESTEC, starting months before a test slot. ESOC performs activities in these test slots with the same ground segment systems as used for flight, resulting in highly representative test results. For AOCS operations preparation, these test slots are used to validate AOCS flight operations procedures and operational products provided by flight dynamics.

In addition, the AOCS S/C operations engineer devotes a large amount of work to the development of the ESOC simulator, ensuring the modeling is flightrepresentative for all AOCS-related aspects.

The Assembly, Integration, and Verification (AIV) schedule for BepiColombo is such that the MPO is in a more mature state earlier, while the full cruise composite including the MTM will only be completed later. Therefore, AOCS operations preparation at ESOC is initially focussed on MPO stand alone, prior to shifting the attention to MCSC configuration, which will have to be operated in the first 7 years of the mission.

\subsection{AOCS Flight Procedures and Flight Dynamics Commanding Interface Implementation Status}

Work on AOCS flight operations procedures started in late 2013 and has progressed significantly in 2014/2015. As for other ESOC missions, flight operations procedures for BepiColombo are written using the MOIS (Manufacturing and Operations Information System) suite of tools [4].

A total number of 290 AOCS procedures is currently expected to be required, out of which 134 have been written, with 78 procedures already run in IGST.

Regarding the commanding interface with flight dynamics, a total of 263 requests have currently been defined, with the interface control document in a stable state. These requests fall into the following categories:

- commanding of AOCS units (e.g., star tracker alignment matrices, IMU calibration settings);

- commanding of AOCS parameters (e.g., mass properties, thruster modulation settings);

- commanding of ephemerides and guidance in random access memory (RAM) and SGM; and

- AOCS mode transitions (e.g., entry to OCM, setting all maneuver parameters as required). 
The commanding requests for uplink of AOCS ephemerides (Sun and Earth) were implemented in late 2013. Requests for guidance updates for MPO configuration were implemented throughout 2014, followed by the same requests for other composite S/C configurations in late 2014 and early 2015. The implementation of essential commanding requests for unit operations (RW management and antennae operations) as well as the implementation of OCM commanding products have started in early 2015 and is planned to be completed prior to IGST-4 in July 2015.

\subsection{AOCS Closed-Loop Testing on the ESOC Simulator}

In late 2013, the ESOC simulator was already capable of running an AOCS closed-loop scenario. As in the early stages of OBSW development, the system initialization process for loading context information from the permanent SGM was not yet ready, the AOCS reference cases were performed by patching AOCS configuration and guidance information directly in OBC RAM. As a result, the setup for these reference test cases was not flight representative.

Therefore, the prime objective in 2014 was to obtain the ability for setting up a closed-loop run based on a custom state vector consisting of epoch, orbital position, S/C attitude, S/C angular rates, with the S/C correctly pointing and having correct guidance information stored in SGM corresponding to this state vector (as for flight). This is a key step for AOCS operations preparation, as it requires preparation of AOCS guidance products as well as a thorough understanding on how the AOCS and, in particular, the AOCS system initialization process works. For interplanetary missions, this can be intricate owing the significant amount of consistent guidance information needed by the AOCS to perform nominally, e.g., knowledge on Earth direction to point antennae correctly or ground-provided orbital data. For BepiColombo, an additional complexity is that the AOCS needs context information on the last known S/C attitude and angular rates when entering safe/survival mode.

The following guidance and ephemeris context is required by the BepiColombo AOCS for functioning correctly following a safe/survival mode entry in MPO configuration:

- last known $S / C$ attitude and angular rates (SGM RAM): as described in section 2 and unlike traditional designs, the AOCS does not perform a Sun search/acquisition using sensor data when entering SASM. Instead, it uses context information stored in SGM RAM at $1 \mathrm{~Hz}$ when the AOCS was running nominally;

- Sun ephemerides (SGM Electrically Erasable Programmable Read-Only Memory (EEPROM)): in the early phases of SASM, Sun pointing is estab- 
lished based on last known attitude, angular rates, and the Sun direction based on ground-provided Sun ephemerides;

- Earth ephemerides (SGM EEPROM): required for pointing the MGA and HGA to the Earth in SHM and higher modes;

- MPO SASM attitude and solar array guidance (SGM EEPROM): the SASM attitude guidance specifies $\mathrm{S} / \mathrm{C}$ rotation rate and phasing for the rotation around the Sun line which has to be in synch with the orbital motion around Mercury in order to ensure that the radiator side of the MPO is never facing Mercury. The SASM SA guidance consists of look-up tables with time ranges and commanded Sun aspect angles (SAA), allowing for a stepwise adjustment of the SA position as the $\mathrm{S} / \mathrm{C}$ rotates around the Sun line; and

- MPO SHM attitude and solar array guidance (SGM EEPROM): SHM is the highest mode reached autonomously after safe/survival mode entry. For MPO, both attitude and SA guidance are specified as Chebyshev polynomials. The default attitude guidance for SHM loaded in SGM EEPROM is such that the attitude profile is the same as in SASM.

In summer 2014, a first successful closed-loop run was achieved on the ESOC simulator, with the procedure involving the following basic steps:

(1) a custom scenario is agreed, allowing flight dynamics to provide the necessary commanding products;

(2) the simulator is started with a configuration in which the AOCS is in SBM;

(3) the last known S/C attitude and angular rates are loaded into SGM RAM, disabling write access beforehand in order to avoid that the information gets overwritten by the AOCS;

(4) SGM EEPROM context is configured including AOCS mode transition settings and other essential context such as declaring that the launcher separation sequence has been terminated;

(5) the AOCS ephemerides and guidance products are prepared for uplink to SGM EEPROM. This requires time shifting the products according to the desired start time of the closed-loop run. As the mission control system is working in coordinated universal time (UTC), yet the guidance products are generated for the future epoch time, a time correlation between these two times has to be maintained carefully to correctly set up the scenario; and 
(6) when the desired start time is reached, a simulator script is started which changes the state vector and commands a reboot of the OBC. Following the reboot, the closed-loop simulation begins, with the OBSW loading the necessary context information from SGM.

In early 2015, an AOCS-closed loop in MCSC configuration was also achieved on the ESOC simulator. In the future, the steps to set up a closed-loop run on the simulator for a new state vector are planned to be simplified by script, allowing to automatically load the products provided by flight dynamics and setting the state vector according to a dedicated input file.

\subsection{Testing Activities on the Spacecraft Engineering Test Bed and Flight Model}

Following successful establishment of a custom scenario for AOCS closed-loop testing on the ESOC simulator, in December 2014, a first AOCS closed-loop run was achieved on the ETB in the frame of the 2-day IGST-3 slot. The purpose of this test slot was to exercise a wide range of AOCS operations activities in modes SASM, SHM, and NM, with the S/C in MPO configuration.

The AOCS closed-loop run on each IGST-3 day was set up based on an ESOCprovided initial state vector. As the setup of a closed-loop run had never been done before with industry, two IGST-3 dry runs were required in November 2014 to sort out the basic procedure and interaction between AIV and ESOC. The closed-loop was set up each day according to a procedure similar to AOCS closedloop testing with the ESOC simulator:

(1) in advance to IGST-3, the state vector was provided to AIV for inclusion in the setup of the AOCS SCOE;

(2) on the testing day, AIV configured the ETB for "Basic Test Mode" with AOCS in SBM;

(3) after TC handover, the context information on S/C attitude and angular rates was loaded into SGM RAM;

(4) ESOC configured various SASM/SHM mode transition settings in SGM EEPROM, governing terminal AOCS mode and transition duration settings;

(5) an UTC time for starting the closed-loop simulation was agreed with AIV. The AOCS guidance products were then shifted from simulation epoch time (e. g., 2024 Mercury orbit case) to UTC, accordingly, by ESOC and uplinked to SGM EEPROM. For uplinking this guidance to the $\mathrm{S} / \mathrm{C}$, it is important to have a correct "time correlation" in the mission control system, i.e., 
the correlation between UTC and S/C onboard time (OBT). A schematic overview on time correlation activities is shown in Fig. 6; and

(6) coordinating on the voice loop, at the agreed time the dynamic simulation was started by AIV in the AOCS SCOE, and ESOC commanded an OBC reboot at the same time.

This setup was successful for the different state vectors used on each of the two days. The ensuing testing activities in $\mathrm{SASM} / \mathrm{SHM} / \mathrm{NM}$ were executed successfully, with only minor problems found regarding the procedures or the OBSW. Figure 7 gives a high-level overview of the IGST-3 test cases. Thanks to the effort spent earlier in 2014, most operations in IGST-3 could be done using actual flight operations procedures, which is unusual for this early stage of operations preparation.

Each IGST-3 test case is designed to address different routine and contingency

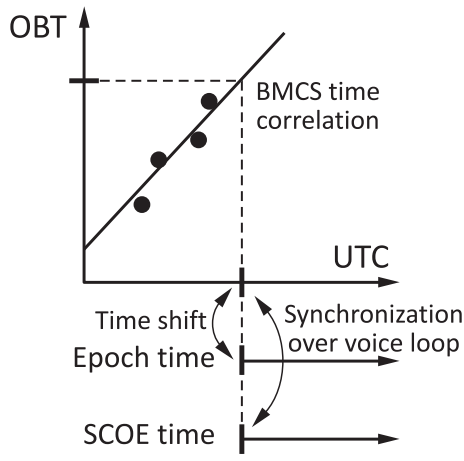

Figure 6 Time correlation in IGST-3 (SCOE - special checkout equipment) operations expected to be performed in flight. An example is the guidance commanding in SASM that addresses a critical part of a survival mode recovery. In a typical survival mode scenario, the $\mathrm{S} / \mathrm{C}$ is expected to be in SASM, with its MGA strobing the Earth in each rotation of the S/C around the Sun line. In such a case, ground would need to be able to stop the S/C rotation, perform small attitude corrections, update the SASM guidance, etc. in order to establish an uninterrupted communication and recover the S/C. Figure 8 shows the operations exercised during the corresponding IGST-3 test case. Figure $8 a$ is the magnitude of the $\mathrm{S} / \mathrm{C}$ angular rate which is expected to be around $2.6 \mathrm{deg} / \mathrm{min}$ for the given test scenario, corresponding to one full revolution of the $\mathrm{S} / \mathrm{C}$ around the Sun line during one Mercury orbit with a period of roughly $138 \mathrm{~min}$. Figure $8 b$ shows the angle between $\mathrm{S} / \mathrm{C}-Z$ axis and the Sun direction, which is kept at $6^{\circ}$ in MPO safe mode guidance. The following operations are visible in these plots:

(1) Up to Epoch+2700 s: by default, the AOCS uses a coarse attitude control deadband $\left( \pm 2.5^{\circ}\right)$ in survival mode to limit fuel consumption, with the SAA drifting within these limits;

(2) Epoch+2700 s: a tighter attitude deadband $\left( \pm 1^{\circ}\right)$ is commanded. In-flight, the resulting more precise $\mathrm{S} / \mathrm{C}$ pointing would increase stability of communications with ground, at the expense of higher fuel consumption; 


\begin{tabular}{|c|c|c|c|}
\hline h & $\min$ & ISGT-3 Day 1 & IGST-3 Day 2 \\
\hline & & ETB setup by ASD & ETB setup by ASD \\
\hline 09 & 00 & \multirow{8}{*}{$\begin{array}{l}\text { ETB handover and start of closed loop simulation } \\
\text { Status at the end of SY-SVT-300: } \\
\text { - AOCS closed loop kicked off } \\
\text { - Valid guidance for SASM, SHM, NM loaded } \\
\text { - ETB configured following nominal reset } \\
\text { - AOCS transitions settings: AOCS stops in SASM-SAPP-3 } \\
\text { (flag STR_USAGE=FALSE), exit SASM to SHM disabled } \\
\text { (STAP_SHM and ESP_SHM are FALSE), autonomous } \\
\text { transition SHM-TCP to SHM-WCP disabled } \\
\text { (shmlsRwTransAlwd = FALSE) } \\
\text { - SSMM packet store reads open }\end{array}$} & \multirow{8}{*}{$\begin{array}{l}\text { ETB handover and start of closed loop simulation } \\
\text { Status at the end of SY-SVT-300: } \\
\text { - AOCS closed loop kicked off } \\
\text { - Valid guidance for SASM, SHM, NM loaded } \\
\text { - ETB configured following nominal reset } \\
\text { - AOCS transition settings: STR_USAGE = TRUE, exit SASM } \\
\text { to SHM enabled (STAP_SHM = TRUE), autonomous } \\
\text { transition SHM-TCP to SHM-WCP disabled } \\
\text { (shmlsRwTransAlwd = FALSE) => terminal mode is SHM- } \\
\text { TCP } \\
\text { - SSMM packet store reads open }\end{array}$} \\
\hline & 15 & & \\
\hline & 30 & & \\
\hline & 45 & & \\
\hline \multirow[t]{4}{*}{10} & 00 & & \\
\hline & 15 & & \\
\hline & 30 & & \\
\hline & 45 & & \\
\hline \multirow[t]{4}{*}{11} & 00 & \multirow{15}{*}{$\begin{array}{l}\text { SASM operations } \\
\text { - FSS activities in SASM-SAPP-3 } \\
\text { - Transition from SASM-SAPP3 to SASM-StAP } \\
\text { - Guidance-related commanding in SASM-ESP } \\
\text { - MGA activities in SASM-ESP } \\
\text { - RW checkout in SASM-ESP } \\
\text { - STR activities in SASM-ESP } \\
\text { - CPS activities in SASM-ESP }\end{array}$} & \multirow{16}{*}{$\begin{array}{l}\text { SHM operations } \\
\text { - Switch on wheels in SHM-TCP and perform transition to } \\
\text { SHM-WCP } \\
\text { - RW activities in SHM } \\
\text { - MGA activities in SHM } \\
\text { - IMU activities in SHM } \\
\text { - Transition from SHM to NM }\end{array}$} \\
\hline & 15 & & \\
\hline & 30 & & \\
\hline & 45 & & \\
\hline \multirow[t]{4}{*}{12} & 00 & & \\
\hline & 15 & & \\
\hline & 30 & & \\
\hline & 45 & & \\
\hline \multirow[t]{4}{*}{13} & 00 & & \\
\hline & 15 & & \\
\hline & 30 & & \\
\hline & 45 & & \\
\hline \multirow[t]{4}{*}{14} & 00 & & \\
\hline & 15 & & \\
\hline & 30 & & \\
\hline & 45 & \multirow{9}{*}{$\begin{array}{l}\text { Operations in SHM-TCP and mode transitions } \\
\text { - Transition from SASM-ESP to SHM-TCP by setting } \\
\text { ESP_SHM to TRUE } \\
\text { - RW checkout in SHM-TCP (leave RW function switched ON) } \\
\text { - Transition from SHM-TCP to SHM-WCP, back to TCP, and } \\
\text { finally again into SHM-WCP } \\
\text { - Transition from SHM to NM }\end{array}$} & \\
\hline \multirow[t]{4}{*}{15} & 00 & & NM operations \\
\hline & 15 & & - Miscellaneous activities in NM (STR, GSE, SADP) \\
\hline & 30 & & \\
\hline & 45 & & \\
\hline \multirow[t]{4}{*}{16} & 00 & & \\
\hline & 15 & & \\
\hline & 30 & & \\
\hline & 45 & & \\
\hline 17 & 00 & Handover to ASD, test debriefing & Handover to ASD, test debriefing \\
\hline
\end{tabular}

Figure 7 Schedule of IGST-3, first AOCS-closed loop testing with the ETB in December 2014

(3) Epoch+3500 s: SASM guidance is updated to introduce about 10 degree offset to the reference attitude around the Sun line which leads to an increase in $\mathrm{S} / \mathrm{C}$ rates. The 10 degree offset chosen in the test case was an example. Similar guidance updates in-flight may be done to adjust for rotational errors in the default SASM guidance used, caused by a drift of the orbit due to thruster usage in survival mode; 


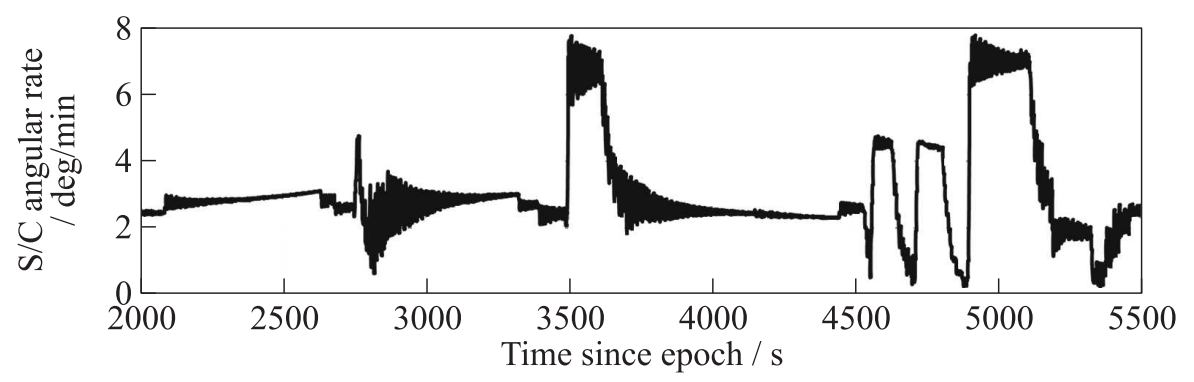

(a)

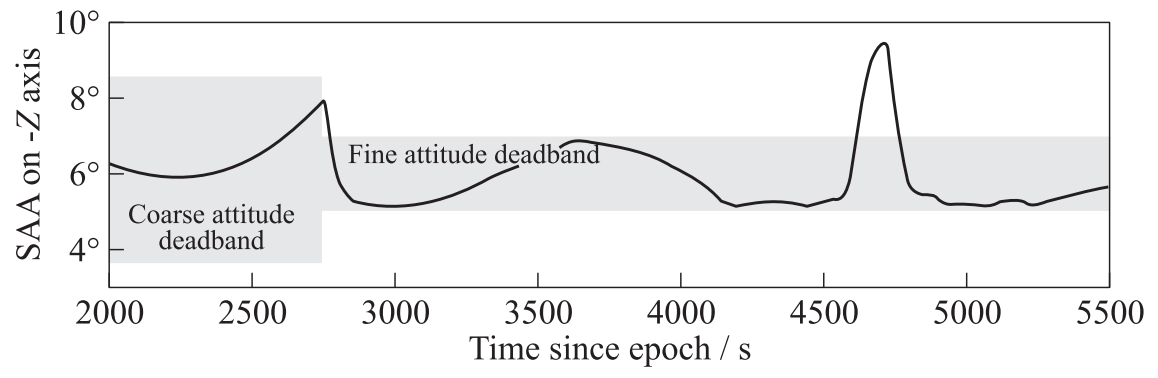

(b)

Figure 8 Spacecraft angular rate and orientation with regard to Sun during SASM guidance commanding on day 1 of IGST-3

(4) Epoch $+4500 \mathrm{~s}$ : rotation around the Sun line is stopped and then an attitude correction is introduced, which results in a 4 degree offset on $\mathrm{S} / \mathrm{C}-Z$ axis, causing the attitude to leave the deadband. The 4 degree offset chosen here was an example. With the rotation stopped, offsets of typically smaller magnitude may be commanded in whatever direction necessary to fine tune MGA pointing to the Earth;

(5) Epoch +4700 s: attitude correction is set back to zero;

(6) Epoch +4900 s: rotation around the Sun line is restarted. Spacecraft rate increases to $7 \mathrm{deg} / \mathrm{min}$ to catch up with the commanded SASM guidance; and

(7) Epoch+5200 s: 10 degree offset is removed which causes the $\mathrm{S} / \mathrm{C}$ rates to stay below $2.6 \mathrm{deg} / \mathrm{min}$ until the new guidance has converged.

Another example for operations tested in IGST-3 are RW activities in SHM, including RW offloading operations and taking an RW out of the AOCS loop. Figure 9 gives the RW unit (RWU) angular momentum profiles and the magni- 

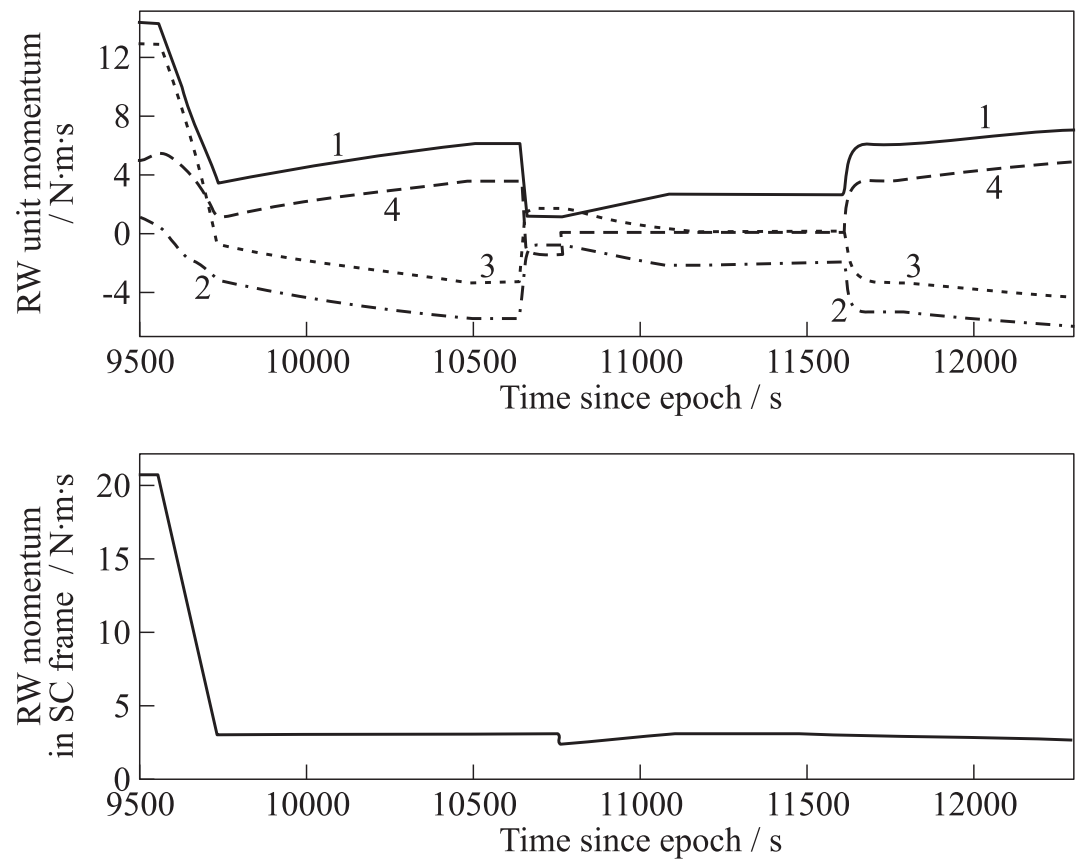

Figure 9 Reaction wheels momentum profiles (1 to 4 ) during RW operations on day 2 of IGST-3

tude of the total angular momentum of the RW cluster. The following operations are performed:

(1) Epoch+9600 s: wheel offloading is commanded to remove the excessive RW cluster momentum;

(2) Epoch+9750 s: wheel offloading is completed successfully;

(3) Epoch +9750 to $10500 \mathrm{~s}$ : the momentum of each wheel keeps increasing although there is no significant change in the total momentum of the RW cluster. This increase is due to the on board null space management, active whenever all 4 wheels are in the loop. The target null space magnitude was set to $7.5 \mathrm{~N} \cdot \mathrm{m} \cdot \mathrm{s}$ by ground for this test scenario; thus, wheel momentums keep drifting until this target is achieved;

(4) Epoch +10500 s: RW4 is taken out of the AOCS torque control loop and commanded in speed mode, i.e., at constant momentum. The null space drift is also stopped with only 3 wheels in the loop; 
(5) Epoch+10700 s: RW4 momentum is changed to $-1.5 \mathrm{~N} \cdot \mathrm{m} \cdot \mathrm{s}$ to see other wheels absorbing the change in its momentum, with the total angular momentum remaining constant;

(6) Epoch $+10800 \mathrm{~s}$ : RW4 is switched off, which creates a jump in the total angular momentum as RW4 is not anymore considered a part of the wheel cluster;

(7) Epoch+11600 s: RW4 is switched on in constant speed mode; and

(8) Epoch +11800 s: RW4 is put back in the AOCS torque control loop.

IGST-3 provided the first opportunity for the FCT to run a wide range of AOCS operations in a realistic context, using the ETB closed-loop capability. Several more such test slots are coming up in the near future. IGST-4 is planned for July 2015 and will contain a test of all possible AOCS modes and unit operations in MPO configuration, including activities not run in IGST-3, the most important of which are orbit control manoeuvres in OCM. Flight dynamics commanding requests are planned to be used more extensively in IGST-4, not only covering AOCS guidance but also unit level operations for RWs and antenna. IGST-4 will also be the first time AOCS closed-loop testing with the FCE in the loop is planned to be done. The very first SVT is then planned for the last quarter of 2015, including a rerun of the AOCS operations activities performed in IGST-4.

\section{CONCLUDING REMARKS AND OUTLOOK}

The 2018 ESA/JAXA BepiColombo mission to Mercury poses a particular challenge for AOCS operations, with the AOCS controlling a highly complex composite $\mathrm{S} / \mathrm{C}$, and strict constraints imposed by the harsh thermal environment leading to complex ground-provided guidance for $\mathrm{S} / \mathrm{C}$ attitude and steerable mechanisms.

AOCS operations preparation at ESA/ESOC is in an advanced state. Generation of flight operations procedures for stand-alone operations of the ESA MPO progressed very well in 2014/2015. Given the mature state of the mission control system and the $\mathrm{S} / \mathrm{C}$ simulator and the ability of flight dynamics to generate essential S/C attitude and solar guidance products, in 2014 the execution of custom AOCS closed-loop scenarios was achieved. Extensive AOCS testing was performed on industry's ETB of the MPO S/C in late 2014, executing the complex collaboration activities between AIV and ESOC required for setting up a closed loop run and performing a wide range of AOCS operations.

In terms of future AOCS operations preparation work, several more tests with the engineering model and the flight model are coming up. Focus will now slowly 
shift to operations preparation for the cruise stack, including electric propulsion operations.

\section{REFERENCES}

1. Benkhoff, J., J. van Casteren, H. Hayakawa, et al. 2010. BepiColombo - comprehensive exploration of Mercury: Mission overview and science goals. Planet. Space Sci. 58(1-2):2-20.

2. Clerigo, I., E. Montagnon, and D. Segneri. 2014. A simulated journey to Mercury: The challenges of the BepiColombo simulator development for the flight control team. SpaceOps Conference. Pasadena, CA.

3. Montagnon, E., P. Ferri, and V. Companys. 2008. Operational challenges of the ESA/JAXA BepiColombo mission. SpaceOps Conference. Heidelberg, Germany.

4. Rhea Group. January 29, 2014. Manufacturing and Operations Information System (MOIS) White Paper. 